\title{
Dynamic Simulation of Crank-group Driving Mechanism with Clearance
}

\author{
http://dx.doi.org/10.3991/ijoe.v12i02.5042 \\ Yansong Liu, Jujiang Cao \\ Shaanxi University of Science and Technology, Xi'an, China
}

\begin{abstract}
The crank-group driving mechanism includes a group of redundant constraints. This mechanism can move as a driving mechanism under ideal conditions, but it may be stuck because of the processing errors in practical applications. So, some clearances are reserved between the pin hole and the pin to ensure that the crank-group driving mechanism can achieve normal movement, but these clearances inevitably affect the dynamic performance of the output member. In this paper, the dynamic simulation model of the crank-group driving mechanism is established by NX/Motion Simulation which is based on the 3-D model of planar link mechanism. The comparative analysis is made about the dynamic characteristics of the mechanism which is influenced by different clearances, different angular velocity, different damping, and make a qualitative analysis how the clearances affects the dynamic performance of the output member. The simulation results also show that appropriately increasing the angular velocity, increasing stiffness and damping of the components can effectively inhibit the adverse influences of clearance on the dynamic characteristics of the mechanism.
\end{abstract}

Index Terms - clearance, crank-group driving mechanism, dynamic simulation

\section{INTRODUCTION}

In some mechanisms, transmitting small torque, or having a larger center distance, such as some special machine, some packaging machinery, some tobacco machinery ,using the crank-group driving mechanism instead of common transmission mode such as gears, chains can simplify the structure and cut the cost [1]. Fig.1 shows the application of the crank-group driving mechanism in the tobacco machinery.

The general form of crank-group driving mechanism is shown in Figure 2. Driving crank and driven cranks have the same phase, structure and size, therefore, driving crank, any driven crank and the rod between them can be seen as a parallelogram mechanism. Under ideal conditions (no size error, no stress deformation, and no installation error), each crank doe's rotary movement around its own axis, and the rod does translational motion around its own center[3]. The redundant constraints are often transformed into statically indeterminate structure because of the errors and material deformation in processing and installation. The appropriate clearance fit between sizes of the crank pin and rod pin hole are selected to avoid statically indeterminate structure and ensure the feasibility of the mechanism motion [4]. But the mechanism's output parameters are adversely affected by the clearances. The

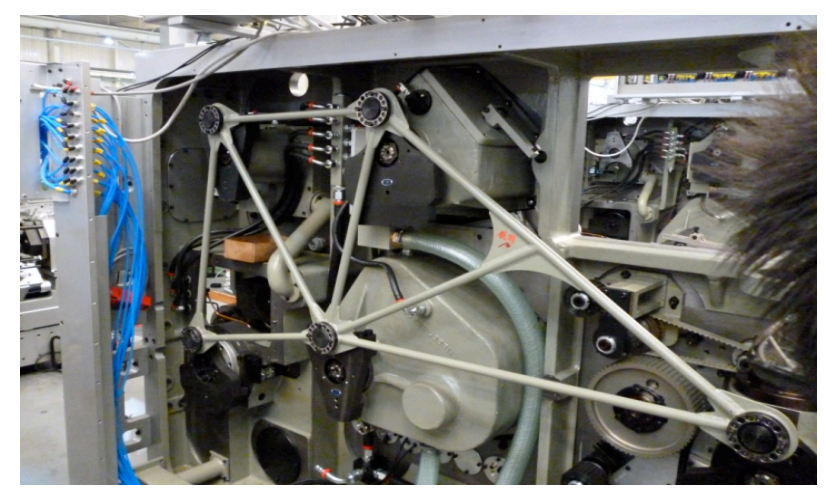

Figure 1. The application of the crank-group driving mechanism in the tobacco machinery

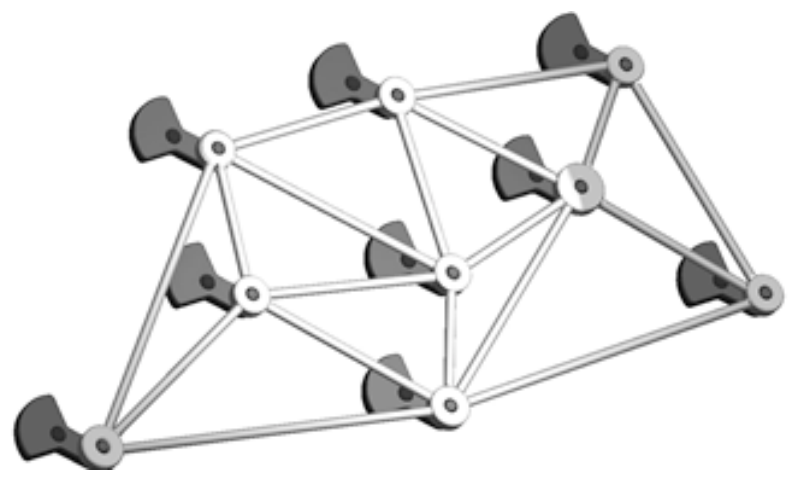

Figure 2. The general form the crank-group driving mechanism

dynamic simulation of the crank-group driving mechanism with clearance can help to grasp the mechanism's dynamic characteristics, know the real motion model of each crank, and design the structure.

\section{Clearance MODEL}

For simplicity, a schematic diagram of the crank-group driving mechanism based on parallelogram mechanism is shown in Fig.3.

$A_{1} B_{1}$ is the drive crank. $A_{2} B_{2}$ and $A_{3} B_{3}$ are the driven cranks. $r$ is the radius of the pin, and $R$ is the radius of the pin hole on $B_{1}$. The collision is caused by the clearance between the pin and the pin hole. The non-linear spring-damper model is used to describe the clearance between the pin and the pin hole, that is, the collision force can be shown by formula 1 when two objects come into contact $[1,2,5]$. 


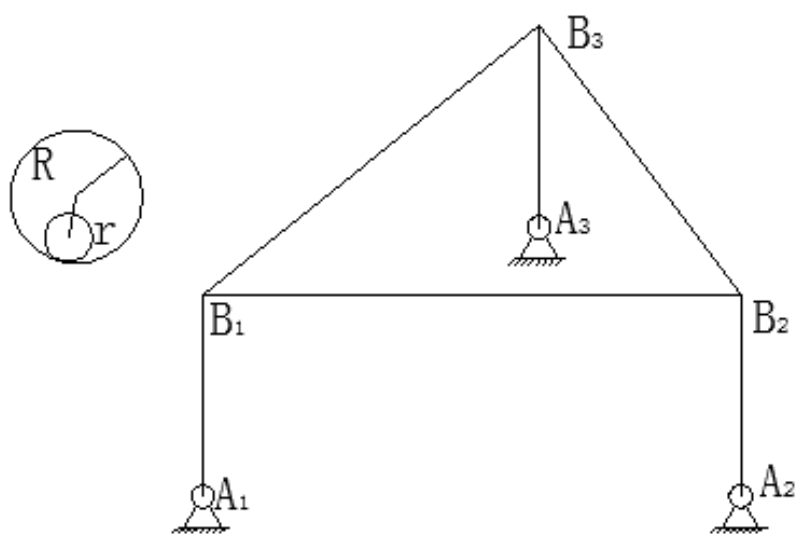

Figure 3. A diagram of the crank-group driving mechanism

$$
\begin{cases}F_{n}=k \delta^{q}+\operatorname{Step}\left(\delta, 0,0, \delta_{\max }, C_{\max }\right) & \delta>0 \\ F_{n}=0 & \delta \leq 0\end{cases}
$$

In the formula $1, F_{n}$ represents the normal force, $q=1.5$ represents the collision index, $\delta$ represents the penetrating amount on the normal direction of the two objects' contact surfaces, $k$ represents equivalent stiffness coefficient, $k=\frac{4}{3} R^{1 / 2} E^{*}, 1 / R=1 / R_{1}+1 / R_{2}, \quad R_{1}, R_{2}$ represent the contact radius of two objects, $\frac{1}{E^{*}}=\frac{1-u_{1}^{2}}{E_{1}}+\frac{1-u_{2}^{2}}{E_{2}}, u_{1}, u_{2}$ represent respectively the Poisson's ratio of two objects, $E_{1}, E_{2}$ represent respectively the elastic modulus of the two objects, $c_{\max }$ represents the maximum damping, $\delta_{\max }$ represents the maximum penetrating amount on the normal direction of the two objects' contact surfaces, ${ }^{v_{\tau}}$ represents the relative velocity on the tangential direction of the two objects' contact surfaces ,and $\operatorname{Step}\left({ }^{*}\right)$ represents the damping function shown by the formula 2 .

$\operatorname{Step}\left(\delta, 0,0, \delta_{\max }, C_{\max }\right)= \begin{cases}0 & \delta \leq 0 \\ C_{\max }\left[\frac{\delta}{\delta_{\max }}\right]^{2}+\left[3-2 \frac{\delta}{\delta_{\max }}\right] & 0 \leq \delta \leq \delta_{\max } \\ C_{\max } & \delta \geq \delta_{\max }\end{cases}$

\section{SIMULATION MODEL OF CRANK-GROUP DRIVING MECHANISM WITH CLEARANCE}

The simulation model shown in Fig. 4 of crank-group driving mechanism with clearance is built on its diagram shown in Fig.3. $A_{1} B_{1}, A_{2} B_{2}, A_{3} B_{3}$ all represent the crank length, and their value are all $300 \mathrm{~mm}$. $B_{1} B_{2}, B_{2} B_{3}, B_{1} B_{3}$ all represent the rod length, and their value are respectively $600 \mathrm{~mm}, 527 \mathrm{~mm}$ and $592 \mathrm{~mm}$. the density of all parts is $7830 \mathrm{~kg} / \mathrm{m} 3$. The nominal diameter

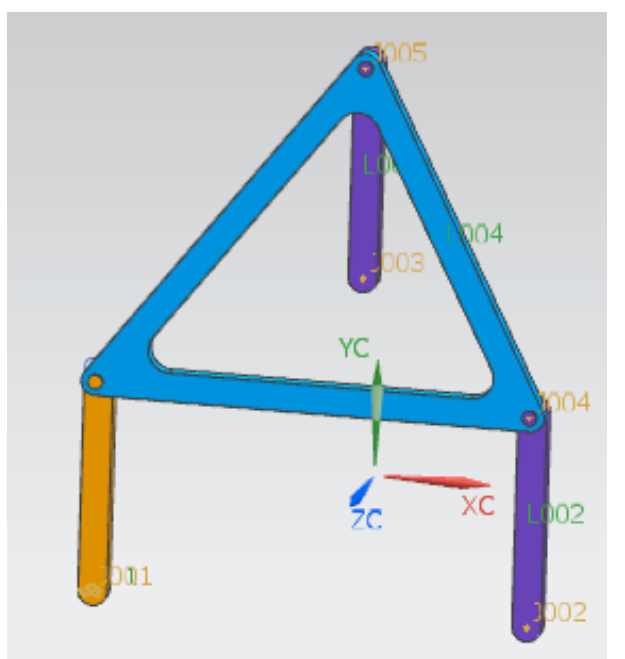

Figure 4. Simulation model of crank-group driving mechanism with clearance

of the pin and pin hole is $\phi 20$. In order to simplify the model, only the clearance at point ${ }^{B_{1}}$ associated with the drive crank $A_{1} B_{1}$ is considered, and the clearance fit is $\phi 20 \mathrm{H} 8 / \mathrm{h} 7$, that is, the clearance maximum is $0.054 \mathrm{~mm}$.

\section{SIMULATION RESULTS AND ANALYSIS}

\section{A. The influence of the clearance on the angular acceleration of the driven crank}

The angular acceleration curves of the driven crank are shown in Figure 5 when the clearance between the pin and pin hole are taken $0.054 \mathrm{~mm}$ and $0.027 \mathrm{~mm}$. From Figure 5 , when the drive crank angular velocity is $600 \mathrm{r} / \mathrm{min}$ and other conditions unchanged, the maximum amplitude of the driven crank angular acceleration when the clearance is $0.054 \mathrm{~mm}$ is much larger than that when the clearance is $0.027 \mathrm{~mm}$.

\section{B. The influence of the angular velocity of drive crank} on the angular acceleration of the driven crank

The angular acceleration curves of the driven crank are shown in Figure 6 when the angular velocities of drive crank are taken $300 \mathrm{r} / \mathrm{min}$ and $600 \mathrm{r} / \mathrm{min}$. From Figure 6, when the clearance is $0.054 \mathrm{~mm}$ and other conditions unchanged, the maximum amplitude of the driven crank angular acceleration when the angular velocity of drive crank is $600 \mathrm{r} / \mathrm{min}$ is much larger than that when the angular velocity is $300 \mathrm{r} / \mathrm{min}$.

\section{The influence of the material damping on the angular} acceleration of the driven crank

The angular acceleration curves of the driven crank are shown in Figure 7 when material dampings are taken $10 \mathrm{~N}$ $\mathrm{s} / \mathrm{mm}$ and $15 \mathrm{~N}-\mathrm{s} / \mathrm{mm}$. From Figure 7 , when the clearance is $0.027 \mathrm{~mm}$ and other conditions unchanged, increasing the material damping can significantly reduce the magnitude of the angular acceleration of the driven crank. 


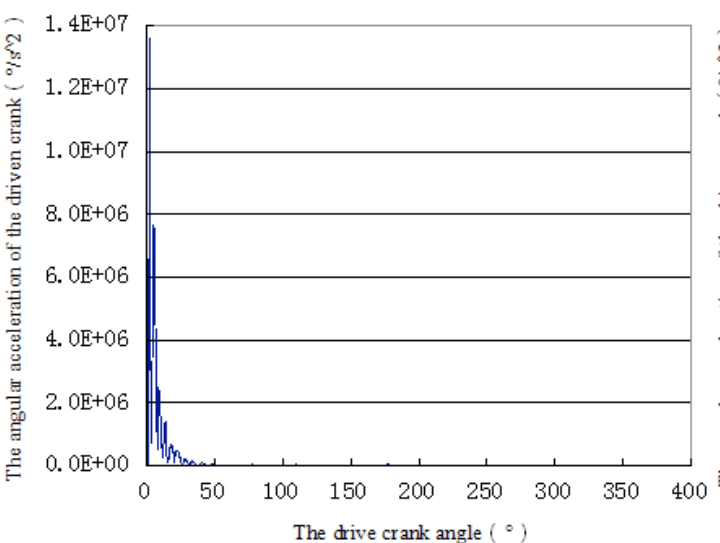

(a) The clearance is $0.054 \mathrm{~mm}$

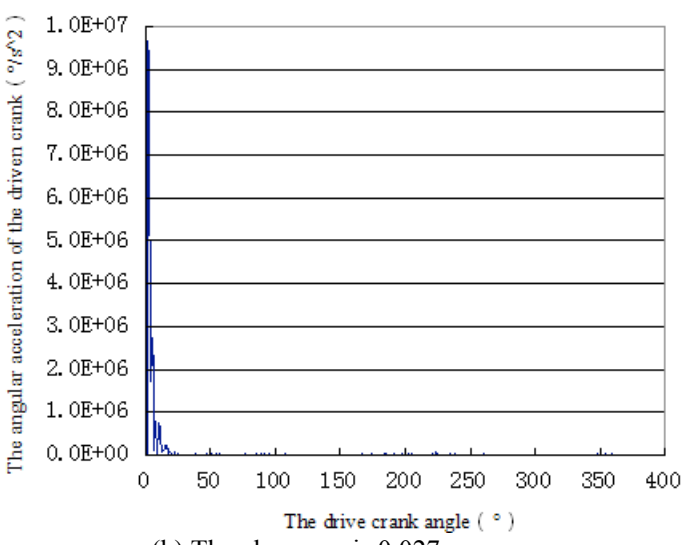

(b) The clearance is $0.027 \mathrm{~mm}$

Figure 5. The angular acceleration curve of the driven crank in different clearances

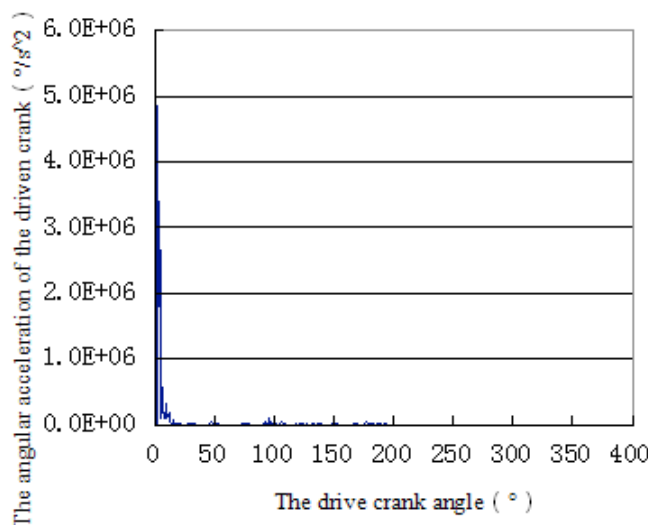

(a) The angular velocity is $300 \mathrm{r} / \mathrm{min}$

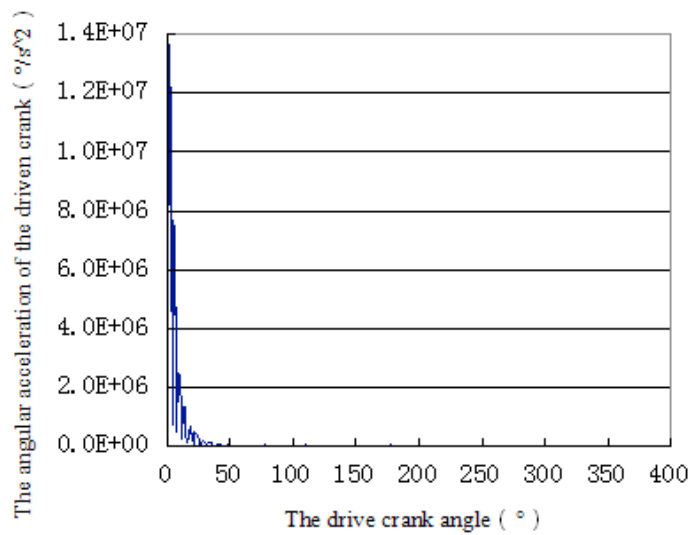

(b) The angular velocity is $600 \mathrm{r} / \mathrm{min}$

Figure 6. The angular acceleration curve of the driven crank in different angular velocity of drive crank
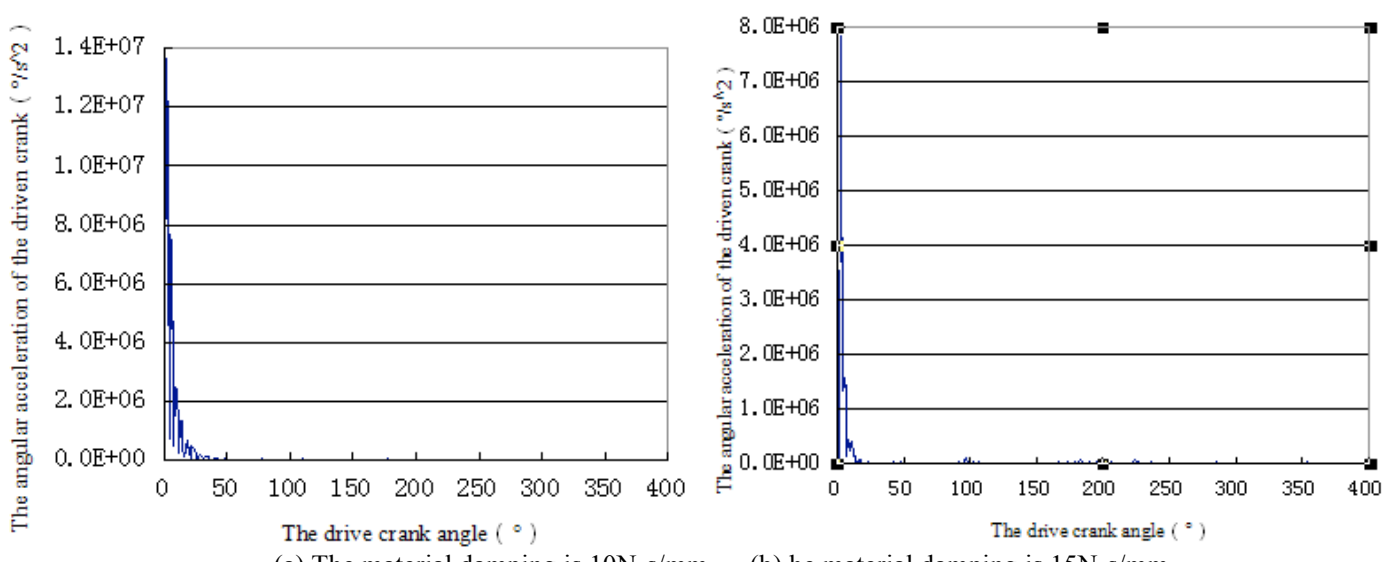

(a) The material damping is $10 \mathrm{~N}-\mathrm{s} / \mathrm{mm}$

(b) he material damping is $15 \mathrm{~N}-\mathrm{s} / \mathrm{mm}$

Figure 7. The angular acceleration curve of the driven crank in different material damping

\section{CONCLUSIONS}

1) The impact caused by the clearance is mainly in the initial phase of the movement, and the influence of the clearance on the angular acceleration of the driven crank is great. But the movement is stable when crank turns to 50 .

2) Increasing angular velocity increases the magnitude of the angular acceleration of the driven crank in the initial phase of the movement, but angular acceleration is relatively stable when the movement stability.
3) Increasing the material damping not only can effectively reduce the angular acceleration magnitude of driven crank in the initial movement, but can further reduce the impact cause by the clearance in the steady phase of the movement.

4) The simulation of the crank-group driving mechanism with clearance based on NX / Motion Simulation can avoid a lot of programming. In the simulation platform, modifying the simulation parameters can achieve the purpose of understanding the movement of the crankgroup driving mechanism with clearances. 
5) The simulation firstly researches the clearances in the crank-group driving mechanism, and hopes to able to find out the some methods improving the dynamic performance of the output member under the premise of the crank-group driving mechanism's normally movement.

6) The qualitative research is made about the influence of the clearances on the dynamic performance of the output member of the crank-group driving mechanism. Some tests will be made under the guidance of the simulation to verify the simulation results.

\section{REFERENCES}

[1] Zhang Jiaji. On Dynamic Modeling of Mechanical Pair Clearance [D]. Xian: Xidian University, 2006.

[2] Guo Xinglin, Zhao Zikun. Dynamic Analysis of a Flexible Crankrocker Mechanism with Clearance [J]. Journal of Mechanical Strength, 2010,32(6):905-909.

[3] Wang Yuqi, Cao Jujiang. Overview of Crank-group Driving Mechanism $[\mathrm{J}]$. Journal of mechanical transmission, 2013,37(4):134-136.
[4] Wang Yuqi, Cao Jujiang. Research on the Clearance in Crankgroup Driving Mechanism [J]. Journal of mechanical transmission,2013,37(7):1-3.

[5] Shi Bing Yuqi, Jin Ye. Dynamic Simulation and Modeling of Revolute Clearance Joint for Virtual Prototyping [J]. Journal of mechanical engineering, 2009, 45 (4): 299-303. http://dx.doi.org/10.3901/JME.2009.04.299

\section{AUTHORS}

Yansong Liu is with College of Light Industry and Energy, Shaanxi University of Science and Technology and College of Mechanical and electrical Engineering, Shaanxi University of Science and Technology, Xi'an, China.

Jujiang Cao College of Mechanical and electrical Engineering, Shaanxi University of Science and Technology , Xi'an, China

This work was supported in part by NSFC(Grant No. 51175313). Submitted 17 September 2015. Published as resubmitted by the authos 23 January 2016. 